\title{
Study on Chronic Low-Grade Inflammation and Influential Factors of Polycystic Ovary Syndrome
}

\author{
Xiangyan Ruan Yujie Dai \\ Department of Endocrinology for Gynecology, Beijing Obstetrics and Gynecology Hospital, Capital Medical \\ University, Beijing, China
}

\begin{abstract}
Key Words
Polycystic ovarian syndrome $\cdot$ Inflammation, low-grade chronic $\cdot$ Insulin resistance $\cdot$ Triglycerides
\end{abstract}

\begin{abstract}
Objective: To determine whether or not there is an increased level of serum C-reactive protein (CRP), of peripheral white cell count and of associated factors in Chinese patients with polycystic ovary syndrome (PCOS). Subjects and Methods: Seventy-four patients with PCOS were recruited for the study. The control group consisted of 51 normal healthy women of a similar age and body mass index (BMI). The basic physical parameters, white cell count, CRP, sex hormone and fasting insulin were measured. The glycolipid metabolic index and the insulin sensitivity index were calculated. Results: The serum CRP level ( 2.13 vs. $0.86 \mathrm{mg} / \mathrm{l}, \mathrm{p}<0.001)$ and total leukocyte count $\left(7.50 \pm 1.83\right.$ vs. $6.55 \pm 1.66 \times 10^{9} /$, $\mathrm{p}<0.01$ ) were significantly higher in PCOS patients than in normal healthy women. Even PCOS patients with normal $B M I$ had an increase in CRP and leukocyte count. The serum CRP level of PCOS patients was positively correlated with BMI $(R=0.57, p<0.001)$ and triglycerides $(R=0.47, p<0.001)$ and negatively correlated with the insulin sensitivity index $(R=$ $-0.60, p<0.001)$. Conclusion: The data showed that chronic nonspecific inflammation exists among Chinese patients with PCOS and that this kind of inflammation was related to insulin resistance, $\mathrm{BMI}$, and triglycerides.
\end{abstract}

Copyright @ 2009 S. Karger AG, Basel
(C) 2009 S. Karger AG, Basel

1011-7571/09/0182-0118\$26.00/0

Fax +4161306 1234

E-Mail karger@karger.ch

www.karger.com
Accessible online at: www.karger.com/mpp

\section{Introduction}

Polycystic ovary syndrome (PCOS) can cause infertility in reproductive-aged women. It is usually complicated by the manifestation of metabolic disorders such as central obesity, insulin resistance (IR), and abnormal blood fat [1]. Recently, it has been shown that PCOS can cause a systemic chronic low-grade inflammation mainly marked by a mild increase in C-reactive protein (CRP) [2]. There is a disagreement about the connection between neuroendocrine dysfunction, especially hyperandrogenism of PCOS patients, and chronic nonspecific inflammation $[3,4]$ based on studies done with Caucasians with PCOS. It has also been shown that there is an ethnic difference in the correlation between androgen and insulin levels [5], glycolipid metabolism [6], and body fat distribution. Li et al. [7] showed that the prevalence of hyperandrogenism, obesity, and IR was lower in Chinese women with PCOS than in women from other ethnic groups. Therefore, it is necessary to find out whether or not chronic low-grade inflammation exists among Chinese patients with PCOS and its influential factors.

Miller et al. [8] reported that chronic low-grade inflammation played an important role during the onset and progression of diabetes and cardiovascular disease. The serum inflammatory factor level can reflect the incidence of these diseases. Blood CRP $<1,1-3$, and $>3$ $\mathrm{mg} / \mathrm{l}$ represent a low, middle, and high risk of cardiovascular disease in the future, respectively. 
The purpose of this study is (1) to discuss whether or not chronic low-grade inflammation exists among Chinese PCOS patients mainly based on the analysis of CRP and peripheral white cell count and (2) to study the correlation between CRP and BMI, central obesity anthropometrical index, testosterone, glycolipid metabolic index, insulin sensitivity index, etc., and determine the factors related to chronic low-grade inflammation of Chinese PCOS patients. This study for the first time evaluated the chronic low-grade inflammation of PCOS patients on the Chinese mainland to provide an experimental foundation for the clinical treatment of PCOS patients and the prevention of long-term complications. The aim of this study was to examine the difference of CRP and peripheral white cell count in women with and without PCOS.

\section{Subjects and Methods}

\section{Subjects}

All PCOS patients attending the Department of Gynecologic Endocrinology, Beijing Obstetrics and Gynecology Hospital, Capital Medical University from July to December 2005 were asked to participate. According to the Rotterdam criteria [9], the diagnosis of PCOS was based on the association of at least two of the three following criteria: (1) oligoovulation or anovulation, (2) clinical or biochemical signs of hyperandrogenism, and (3) polycystic ovaries. Congenital adrenal hyperplasia, androgen-secreting tumors, Cushing's syndrome, thyroid disease and prolactinoma were excluded. The controls were simultaneously recruited from patients seen for an annual exam during the same period. All controls had regular menstrual cycles (at least 12/year) and no history of hirsutism or infertility. The exclusion criteria were women taking drugs such as metformin and oral contraceptives in the 3 months before selection, severe disease of important organs such as heart, liver, lung, kidney, spleen and the hematological system, acute/chronic infectious diseases, trauma, or a history of surgery 1 month before selection; women known to have cardiovascular diseases and/or diabetes; women with blood pressure $>130 / 85 \mathrm{~mm} \mathrm{Hg}$ or hypertension on maintenance medication, severe or multiple thrombosis risk factors, prodrome, or relevant histories, thyroid dysfunction and nicotine and alcohol addiction. All patients gave informed consent before their inclusion in the study. The study was approved by the Human Ethics Committee of the Beijing Obstetrics and Gynecology Hospital, Capital Medical University. A total of 74 PCOS patients and 51 controls were recruited. The control group was similar in age and BMI to the PCOS group.

\section{Design}

Basic Physical Parameters and Blood Index

On the day of the study, subjects reported in the morning in a fasting state to the Department of Endocrinology for Gynecology. Body weight and height were measured to the nearest $0.1 \mathrm{~kg}$ with the patient wearing light clothes and no shoes. The BMI was cal- culated as kilograms per square meter. Waist circumferences (WC) and hip circumferences (HC) were measured in duplicate at the level of the umbilicus (waist) and greater trochanter (hip). Mean systolic and diastolic blood pressure was assessed with two readings after $5 \mathrm{~min}$ of rest sitting down. Ultrasound tests were performed on all the PCOS patients and controls.

The following blood indices of the PCOS patients and the controls group were measured: TSH, prolactin, progesterone, total testosterone, luteinizing hormone $(\mathrm{LH})$, and follicle-stimulating hormone (FSH), estradiol $\left(\mathrm{E}_{2}\right)$, insulin (immunochemiluminescent method, reagents produced by Bayer, Germany), total cholesterol, triglycerides, HDL cholesterol (immunochemistry colorimetry method, reagents produced by Beijing Laibang Company, China), low-density cholesterol, apolipoprotein A1, apolipoprotein B (immunoturbidimetry method, reagents produced by Beijing Laibang Company, China) and CRP (latex agglutination turbidimetry method, reagents produced by Deling Company, China). Oral glucose intolerance was performed in all subjects with a fasting glucose level followed by a 2-hour glucose level (glucose oxidase oxygen electrode method, reagents produced by Shanghai Fenghui Medical Science and Technology Company, China) obtained after a 75-gram glucose load. White cell count in endcapillary blood, namely finger blood, was determined with the blood analyzer. All subjects had regular diets on the 3 days before drawing blood. Blood samples were taken from those with regular menses $(n=54)$ during the 3rd to 5th day of the menstrual cycle and from those with irregular menses $(n=71)$ after a transvaginal ultrasound that revealed no follicles of a diameter $>10 \mathrm{~mm}$.

The homeostatic model assessment-insulin resistance (HOMA-IR) was used as a surrogate measure of insulin sensitivity, calculated as: fasting insulin $(\mathrm{mU} / \mathrm{l}) \times$ fasting glucose $(\mathrm{mmol} / \mathrm{l}) / 22.5[10]$.

\section{Statistical Analysis}

The SPSS 12.0 (SPSS Institute, Chicago, Ill., USA) statistical software for data management and analysis was used. First, we performed a normality test of data. Data in normal distribution were presented as means $\pm \mathrm{SD}$, while the nonnormal distribution was described with the median. Wilcoxon rank-sum test was used to evaluate differences between PCOS and control groups. The $\chi^{2}$ test was performed for categorical variables. The natural logarithm was used to linearize the insulin sensitivity index and CRP of the group before statistical analysis. The correlation between CRP of the PCOS group and BMI, central obesity anthropometric index, testosterone, glycolipid metabolic index, insulin sensitivity index, FSH and LH indices was analyzed through multiple regression. In all analyses, a two-tailed $\alpha$ level of 0.05 was used.

\section{Results}

Physical and blood indices were measured in all the subjects. Baseline characteristics are shown in table 1. There were no differences between subjects with and without PCOS for age and BMI; however, WC and WC/ $\mathrm{HC}$ ratio were significantly higher in the PCOS group than in the control group $(\mathrm{p}<0.01$ and $\mathrm{p}<0.05$, respec- 
Table 1. Comparison of body measurement indices, blood fat, blood glucose, insulin sensitivity index and inflammation markers in the PCOS group and the control group

\begin{tabular}{lccc}
\hline & $\begin{array}{c}\text { PCOS group } \\
(\mathrm{n}=74)\end{array}$ & $\begin{array}{c}\text { Control group } \\
(\mathrm{n}=51)\end{array}$ & $\mathrm{p}$ \\
\hline Age, years & $28 \pm 4$ & $29 \pm 4$ & 0.115 \\
BMI & $26.49 \pm 4.83$ & $25.33 \pm 3.65$ & 0.130 \\
Waist circumference, cm & $86.6 \pm 11.3$ & $79.9 \pm 9.4$ & $<0.01$ \\
Waist-hip ratio & $0.92 \pm 0.50$ & $0.90 \pm 0.05$ & $<0.05$ \\
Waist circumference/ & & & \\
$\quad$ body height & $0.54 \pm 0.07$ & $0.50 \pm 0.06$ & $<0.001$ \\
Testosterone, ng/dl & $70.95 \pm 23.29$ & $38.95 \pm 10.69$ & $<0.001$ \\
LH, mIU/ml & $10.09 \pm 5.10$ & $4.07 \pm 4.34$ & $<0.001$ \\
FSH, mIU/ml & $5.13 \pm 1.58$ & $4.74 \pm 1.51$ & 0.174 \\
Total cholesterol, mmol/1 & $4.80 \pm 1.01$ & $4.87 \pm 1.18$ & 0.716 \\
Triglycerides, mmol/l & $1.36 \pm 0.84$ & $1.00 \pm 0.58$ & $<0.05$ \\
HDL, mmol/l & $1.25 \pm 0.37$ & $1.45 \pm 0.35$ & $<0.05$ \\
LDL, mmol/l & $3.00 \pm 0.93$ & $2.99 \pm 1.11$ & 0.982 \\
Apolipoprotein A1, g/l & $0.98 \pm 0.27$ & $1.29 \pm 0.40$ & $<0.001$ \\
Apolipoprotein B, g/l & $0.86 \pm 0.23$ & $0.89 \pm 0.25$ & 0.449 \\
Fasting insulin, mU/l & $20.44 \pm 6.83$ & $11.85 \pm 4.46$ & $<0.001$ \\
Fasting glucose, mmol/l & $5.35 \pm 0.60$ & $5.18 \pm 0.49$ & 0.103 \\
2-hour glucose, mmol/l & $6.99 \pm 2.09$ & $6.03 \pm 2.14$ & $<0.05$ \\
HOMA-IR & 4.67 & 2.51 & $<0.001$ \\
CRP, mg/l & 2.13 & 0.86 & $<0.001$ \\
White cell count, $\times 10^{9} / 1$ & $7.50 \pm 1.83$ & $6.55 \pm 1.66$ & $<0.01$ \\
\hline
\end{tabular}

Table 2. Percentage of the PCOS group and the control group in the different CRP ranges

\begin{tabular}{lll}
\hline CRP, $\mathrm{mg} / \mathrm{l}$ & PCOS $(\mathrm{n}=74)$ & Control $(\mathrm{n}=51)$ \\
\hline$<1$ & $40.8 \%(29)$ & $68.7 \%(35)^{*}$ \\
$1-3$ & $32.5 \%(23)$ & $27 \%(14)^{*}$ \\
$>3$ & $27 \%(19)$ & $4.3 \%(2)^{*}$ \\
\hline \multicolumn{2}{c}{$* \mathrm{p}<0.05}$. & \\
\hline
\end{tabular}

tively). The following blood indices were higher in the PCOS than the control group: testosterone, LH $(\mathrm{p}<$ $0.001)$, triglycerides $(\mathrm{p}<0.05)$, fasting insulin $(\mathrm{p}<0.001)$, HOMA-IR ( $<<0.001)$ and 2-hour glucose after a 75gram glucose load $(\mathrm{p}<0.05)$. However, the high-density lipoprotein $(\mathrm{p}<0.05)$ and apolipoprotein $\mathrm{A} 1(\mathrm{p}<0.001)$ were lower in the PCOS group than in the control group. There was no statistical difference between the PCOS and the control group in FSH, LDL and apolipoprotein $\mathrm{B}$. The inflammation marker CRP $(p<0.001)$ and total leuko- cyte count $(\mathrm{p}<0.01)$ were significantly higher in the PCOS group.

The percentage of cases with CRP 1-3 mg/l and CRP $>3 \mathrm{mg} / \mathrm{l}$ was higher in the PCOS group than in the control group (table 2). Twenty-seven percent (19/74) of PCOS patients had CRP $>3 \mathrm{mg} / \mathrm{l}$. BMI, triglycerides, and insulin sensitivity index were the determinants of the CRP increase in PCOS patients. The CRP were positively correlated with BMI $(\mathrm{r}=0.57, \mathrm{p}<0.001)$ and triglycerides $(\mathrm{r}=0.47, \mathrm{p}<0.001)$. The insulin sensitivity index was highly and negatively correlated with CRP $(r=-0.60$, $\mathrm{p}<0.001)$. Total $\mathrm{R}^{2}$ of $\mathrm{BMI}$, insulin sensitivity index, triglycerides $=0.437$. Standard coefficients of insulin sensitivity index, BMI, and triglycerides were $-0.310,0.268$ and 0.208 , respectively. The above three indices accounted for $43.7 \%$ of the cause of CRP increase in the following order: insulin sensitivity index $>\mathrm{BMI}>$ triglycerides. Indices such as WC, WC/HC, and WC/body height for central obesity evaluation were not determining factors of the CRP increase in PCOS patients. Fasting insulin, blood glucose, total cholesterol, high-density lipoprotein, lowdensity lipoprotein, apolipoprotein A1, and apolipoprotein $\mathrm{B}$ also had no direct relationship with CRP increase in this study.

\section{Discussion}

We have shown for the first time that chronic lowgrade inflammation exists in Chinese PCOS patients from the Chinese mainland. The increase in low-grade chronic inflammation in women with PCOS is associated with IR, BMI and triglycerides rather than PCOS status per se. The $27.0 \%$ of PCOS patients with CRP $>3 \mathrm{mg} / \mathrm{l}$ and a higher total leukocyte count than the controls indicate that the PCOS patients were at a high risk of cardiovascular diseases and diabetes.

This study also demonstrated that total testosterone and LH were higher in PCOS patients with neuroendocrine dysfunction than in the control group. Although there was no difference in BMI in the two groups, WC and waist-hip ratio were higher in the PCOS group than in the control group, indicating that PCOS patients are more prone to central obesity. Triglycerides increased significantly while high-density lipoprotein and apolipoprotein A protecting against cardiovascular diseases dropped in the PCOS group, indicating that PCOS patients are more prone to abnormal blood fat than healthy women of a similar age and BMI. The higher fasting insulin level, the higher 2-hour glucose after a 75-gram glu- 
cose load and the higher HOMA-IR in the PCOS demonstrated that PCOS patients with abnormal glucose metabolism were more prone to IR than the control group. Therefore, abnormal glycolipid metabolism is a risk factor for cardiovascular diseases and diabetes in PCOS patients.

In this study, the insulin sensitivity index and BMI were two determining factors of serum CRP level in PCOS patients. CRP was positively correlated with BMI and negatively correlated with the insulin sensitivity index. This conclusion is similar to that of other studies [11, 12], indicating that chronic low-grade inflammation in PCOS patients is mainly caused by abnormal metabolism. Obesity, chronic low-grade inflammation, and IR are closely related to each other [13]. Higher BMI usually means higher total body fat content. Both lipocytes and macrophages in fatty tissue can secrete a proinflammatory factor. Excessive fatty tissue will lead to excessive secretion of proinflammatory factor, resulting in chronic low-grade inflammation, and promoting onset and progression of IR [14]. Triglycerides are another determining factor of the serum CRP level in PCOS patients; CRP increases with increasing triglyceride concentrations. It might be a factor distinguishing Chinese PCOS patients from Caucasians with chronic low-grade inflammation in PCOS. Ahmad et al. [15] reported that in a healthy population with normal BMI, triglycerides between 100 and $150 \mathrm{mg} / \mathrm{dl}(1.13-1.70 \mathrm{mmol} / \mathrm{l})$ were positively correlated with CRP $(>2.1 \mathrm{mg} / \mathrm{l})$. On one hand, triglycerides can cause stress to the endoplasmic reticulum of fatty tissue to generate more inflammatory factor; on the other hand, triglycerides can also cause chronic inflammation of vascular endothelial cells. In human blood circulation, triglyceride-rich lipoproteins are chylomicron and verylow-density lipoprotein. Kawakami et al. [16] reported that very-low-density lipoprotein could activate blood vessel endothelium nuclear factor kappa B. Nuclear factor kappa $B$ is the key nuclear factor for priming and regulating inflammation. We still need more basic and clinical research to prove the correlation between triglycerides and CRP in PCOS patients.

There is still a dispute over whether or not chronic low-grade inflammation is closely related to central obesity $[2,17,18]$. The study of Diamanti-Kandarakis et al. [2] on 25 young Greek women with PCOS and 25 controls of a similar age and body mass index demonstrated that the presence of increased serum levels of inflammation in young women with PCOS appeared to be interrelated with hyperandrogenemia in this insulin-resistant population. The study of Puder et al. [17] on 20 Swiss patients

Low-Grade Chronic Inflammation of Polycystic Ovarian Syndrome with PCOS and 15 controls, matched for body mass index and age, showed that the increase in low-grade chronic inflammation in women with PCOS was primarily associated with increased central fat excess rather than the PCOS status per se. Our study found no correlation between CRP and WC, waist-hip ratio, and WC/body height of PCOS patients. The sample size or race might be the cause of these differences. Our study using linear regression to simultaneously analyze the linear correlation between a dependent variable and multiple independent variables demonstrated that only BMI, triglycerides, and insulin sensitivity index were related to CRP, indicating that the influence of WC on CRP was not significant compared with the three indices. It has been shown that waist-hip ratio, WC/body height, and especially WC were positively correlated with abdominal fat accumulation and can accurately reflect the level of central obesity [19]. Most PCOS patients have central obesity. Therefore, measuring WC alone may fail to accurately reflect the total fat in Chinese PCOS patients. At present, mainly western countries conduct CRP and central obesity studies on Caucasian women. Because fat is distributed in various parts of the body, ethnic differences may exist [20]; hence, more research data are needed on other ethnic groups including Chinese to establish the relationship between various body compositions and chronic low-grade inflammation in patients with PCOS.

Similar to Möhlig et al. [3], we did not find any correlation between serum total testosterone and CRP, BMI, or WC. Although hyperandrogenism is one feature of PCOS, only free testosterone can enter cells and tissue via blood to exert biological functions [21]. Free androgen index (FAI), called total testosterone/sex hormone-binding globulin, is also a common index for evaluating free testosterone. Diamanti-Kandarakis et al. [2] reported that FAI was highly correlated with serum CRP of PCOS patients, which was also found in healthy middle-aged women [22] and postmenopausal women very susceptible to cardiovascular disease [23].

BMI, triglycerides, and IR accounted for a CRP increase of only $43.7 \%$ in PCOS patients. There still remain influential factors of over $50 \%$ not listed in this study. Among these possible factors are IL-6, FAI, CRP, genovariation [24], and exercise habits [25].

\section{Limitations of this Study}

In this study we did not measure the level of sex hormone, haptoglobulin and body fat and could not evaluate the influence of body composition and free testosterone on chronic low-grade inflammation of PCOS patients. 


\section{Conclusion}

The data showed that CRP, total leukocyte count and HOMA-IR were highly significant in PCOS patients compared to healthy control women. Therefore, chronic low-grade inflammation exists among Chinese PCOS patients. This kind of inflammation is related with IR, $\mathrm{BMI}$, and triglycerides; PCOS patients are a high-risk group for cardiovascular diseases and diabetes. Further- more, according to this study, CRP may be a better marker of inflammation in women with PCOS because its evaluation is simple and economical.

\section{Acknowledgement}

This study was supported by the Beijing New Star Project Fund, Ke Xing (2000) No. 95; Natural Science Foundation of Beijing (3042009).

\section{References}

1 Essah PA, Wickham EP, Nestler JE: The metabolic syndrome in polycystic ovary syndrome. Clin Obstet Gynecol 2007;50:205225.

-2 Diamanti-Kandarakis E, Alexandraki K, Piperi C, Protogerou A, Katsikis I, Paterakis T, Lekakis J, Panidis D: Inflammatory and endothelial markers in women with polycystic ovary syndrome. Eur J Clin Invest 2006; 36:691-697.

-3 Möhlig M, Spranger J, Osterhoff M, Ristow M, Pfeiffer AF, Schill T, Schlösser HW, Brabant G, Schöfl C: The polycystic ovary syndrome per se is not associated with increased chronic inflammation. Eur J Endocrinol 2004;150:525-532.

4 Mayes JS, Watson GH: Direct effects of sex steroid hormones on adipose tissues and obesity. Obes Rev 2004;5:197-216.

5 Kitabchi AE, Imseis RE, Bush AJ, WilliamsCleaves B, Pourmotabbed G: Racial differences in the correlation between gonadal androgens and serum insulin levels. Diabetes Care 1999;22:1524-1529.

6 Kauffman RP, Baker VM, DiMarino P, Castracane VD: Hyperinsulinemia and circulating dehydroepiandrosterone sulfate in white and Mexican American women with polycystic ovary syndrome. Fertil Steril 2006;85: 1010-1016.

7 Li L, Yang D, Chen X, Chen Y, Feng S, Wang L: Clinical and metabolic features of polycystic ovary syndrome. Int J Gynaecol Obstet 2007;97:129-134.

8 Miller M, Zhan M, Havas S: High attributable risk of elevated C-reactive protein level to conventional coronary heart disease risk factors: the Third National Health and $\mathrm{Nu}$ trition Examination. Arch Intern Med 2005, 165:2063-2068

-9 The Rotterdam ESHRE/ASRM-Sponsored PCOS consensus workshop group: Revised 2003 consensus on diagnostic criteria and long-term health risks related to polycystic ovary syndrome. Hum Reprod 2004;19:4147.
10 Matthews DR, Hosker JP, Rudenski AS, Naylor BA, Treacher DF, Turner RC: Homeostasis model assessment: insulin resistance and $\beta$-cell function from fasting plasma glucose and insulin concentrations in man. Diabetologia 1985;28:412-419.

11 Boulman N, Levy Y, Leiba R, Shachar S, Linn $\mathrm{R}$, Zinder O, Blumenfeld Z: Increased C-reactive protein levels in the polycystic ovary syndrome: a marker of cardiovascular disease. J Clin Endocrinol Metab 2004;89:2160 2165.

12 Kelly CC, Lyall H, Petrie JR, Gould GW, Connell JM, Sattar N: Low grade chronic inflammation in women with polycystic ovarian syndrome. J Clin Endocrinol Metab 2001;86:2453-2455.

13 Guerre-Millo M: Adipose tissue secretory function: implication in metabolic and cardiovascular complications of obesity. J Soc Biol 2006;200:37-43.

14 Berg AH, Scherer PE: Adipose tissue, inflammation, and cardiovascular disease. Circ Res 2005;96:939-949.

15 Ahmad I, Zhan M, Miller M: High prevalence of C-reactive protein elevation with normal triglycerides $(100-149 \mathrm{mg} / \mathrm{dL})$ : are triglyceride levels below $100 \mathrm{mg} / \mathrm{dL}$ more optimal in coronary heart disease risk assessment? Am J Med Sci 2005;329:173-177.

16 Kawakami A, Aikawa M, Nitta N, Yoshida M, Libby P, Sacks FM: Apolipoprotein CIIIinduced THP-1 cell adhesion to endothelial cells involves pertussis toxin-sensitive $G$ protein- and protein kinase $\mathrm{C}$ alpha-mediated nuclear factor-kappaB activation. Arterioscler Thromb Vasc Biol 2007;27:219-225.

17 Puder JJ, Varga S, Kraenzlin M, De Geyter C, Keller U, Müller B: Central fat excess in polycystic ovary syndrome: relation to low-grade inflammation and insulin resistance. J Clin Endocrinol Metab 2005;90:6014-6021.

18 Connelly PW, Hanley AJ, Harris SB, Hegele RA, Zinman B: Relation of waist circumference and glycemic status to C-reactive protein in the Sandy Lake Oji-Cree. Int J Obes Relat Metab Disord 2003;27:347-354.
19 Snell-Bergeon JK, Hokanson JE, Kinney GL, Dabelea D, Ehrlich J, Eckel RH, Ogden L, Rewers $\mathrm{M}$ : Measurement of abdominal fat by CT compared to waist circumference and $\mathrm{BMI}$ in explaining the presence of coronary calcium. Int J Obes Relat Metab Disord 2004; 28:1594-1599.

20 Albu JB, Kovera AJ, Allen L, Wainwright M, Berk E, Raja-Khan N, Janumala I, Burkey B, Heshka S, Gallagher D: Independent association of insulin resistance with larger amounts of intermuscular adipose tissue and a greater acute insulin response to glucose in African American than in white nondiabetic women. Am J Clin Nutr 2005;82:1210-1217.

21 Blight LF, Judd SJ, White GH: Relative diagnostic value of serum non-SHBG-bound testosterone, free androgen index and free testosterone in the assessment of mild to moderate hirsutism. Ann Clin Biochem 1989;26:311-316.

22 Sowers MR, Jannausch M, Randolph JF, McConnell D, Little R, Lasley B, Pasternak R, Sutton-Tyrrell K, Matthews KA: Androgens are associated with hemostatic and inflammatory factors among women at the midlife. J Clin Endocrinol Metab 2005;90:60646071.

23 Joffe HV, Ridker PM, Manson JE, Cook NR, Buring JE, Rexrode KM: Sex hormone-binding globulin and serum testosterone are inversely associated with C-reactive protein levels in postmenopausal women at high risk for cardiovascular disease. Ann Epidemiol 2006; 16:105-112.

24 Wang Q, Hunt SC, Xu Q, Chen YE, Province MA, Eckfeldt JH, Pankow JS, Song Q: Association study of CRP gene polymorphisms with serum CRP level and cardiovascular risk in the NHLBI Family Heart Study. Am J Physiol Heart Circ Physiol 2006;291:H2752H2757.

25 Nicklas BJ, You T, Pahor M: Behavioural treatments for chronic systemic inflammation: effects of dietary weight loss and exercise training. CMAJ 2005;172:1199-1209. 